\title{
Réponse à la réplique de Roger Levasseur et Yvan Rousseau
}

\section{Ronald Rudin}

Volume 50, numéro 1, été 1996

URI : https://id.erudit.org/iderudit/305504ar

DOI : https://doi.org/10.7202/305504ar

Aller au sommaire du numéro

Éditeur(s)

Institut d'histoire de l'Amérique française

ISSN

0035-2357 (imprimé)

1492-1383 (numérique)

Découvrir la revue

Citer ce compte rendu

Rudin, R. (1996). Compte rendu de [Réponse à la réplique de Roger Levasseur et Yvan Rousseau]. Revue d'histoire de l'Amérique française, 50(1), 135-137.

https://doi.org/10.7202/305504ar d'utilisation que vous pouvez consulter en ligne.

https://apropos.erudit.org/fr/usagers/politique-dutilisation/ 


\section{Réponse de Ronald Rudin à Roger Levasseur et Yvan Rousseau}

Il est évident que Roger Levasseur et Yvan Rousseau ont été dérangés par mon compte rendu de leur ouvrage. Pour ma part, je n'ai pas été troublé outre mesure par leur réponse, à la fin de laquelle ils lancent un appel pour «un véritable débat sur l'histoire des caisses au Québec», débat qui aurait dû avoir lieu il y a longtemps. En fait, en lisant leur réponse, je me demandais pourquoi ils n'avaient pas profité de leur livre pour ouvrir un tel débat. J'ai souligné dans mon compte rendu qu'ils avaient été peu explicites au sujet de la signification de leurs travaux, c'est-à-dire qu'ils n'avaient pas opposé leurs propres conclusions avec celles d'auteurs antérieurs (dont je fais partie) et n'avaient pas suffisamment fait ressortir la signification des enjeux qu'ils avaient décrits. S'ils sont maintenant intéressés à assumer cette responsabilité que je considère essentielle à toute démarche scientifique, alors je suis content d'avoir joué un rôle dans ce processus. 
Toutefois, je voudrais profiter de l'occasion pour faire une mise au point au sujet de certains aspects discutés dans leur réponse. Commençons par la question de leur relation avec le Mouvement Desjardins. Il me semblait normal pour un critique de faire remarquer que les auteurs avaient reçu un appui financier de la part de l'institution à l'étude. Levasseur et Rousseau sont peut-être ennuyés par l'attention que j'ai portée à ce sujet, mais il demeure qu'ils n'étaient pas complètement indépendants de leur sujet d'étude. Alors que leur livre mentionnait qu'ils avaient reçu «un appui financier substantiel» (p. 9) du Mouvement Desjardins, nous apprenons maintenant que «seulement» $15 \%$ des fonds provenaient du Mouvement Desjardins. En pensant à ma recherche sur l'histoire des banques au Québec, je me demande ce que les auteurs de comptes rendus auraient écrit si «seulement» $15 \%$ de mes subventions avaient été versées par les banques...

Poursuivons sur la question des liens avec le Mouvement Desjardins. Les auteurs ont fourni quelques éclaircissements au sujet du «comité de lecture», composé de gens du Mouvement, qui a évalué leur livre. Dans mon compte rendu, je m'étais demandé quel rôle pouvait jouer un tel comité. Je ne pouvais que penser qu'il avait été mis sur pied par le Mouvement, ce qui me semblait plausible en l'absence de précisions de la part de Levasseur et Rousseau. Maintenant, ils nous disent que le comité était le fruit de leur initiative. Quant à savoir pourquoi ils souhaitaient un tel comité, je suis un peu perplexe. Si j'avais été à leur place, j'aurais craint de donner à ces dirigeants la possibilité d'influencer, même subtilement, mes conclusions. Il est évident que je suis plus préoccupé par le maintien d'une apparence d'autonomie du chercheur universitaire qu'ils ne le sont.

Dans un autre ordre d'idées, la réplique des auteurs a aussi fourni certains éclaircissements sur leurs efforts à peine voilés de taire l'identité des directeurs de certaines caisses. Je ne pouvais pas comprendre pourquoi ils avaient fait référence à un de ces directeurs comme Omer B. de la Caisse de Z. alors qu'il était possible de trouver son vrai nom en consultant les notes infrapaginales. Ils nous disent maintenant qu'ils avaient choisi d'éviter de citer des noms afin de donner une portée plus générale à l'expérience de ces directeurs. Ils voulaient, pour reprendre leurs mots, «éviter de singulariser les événements, les lieux et les individus pour mettre l'accent sur les phénomènes sociaux». J'espère qu'on me pardonnera de ne pas avoir réalisé que ceci était leur intention, puisque cette explication n'était pas fournie dans leur livre.

Leurs éclaircissements sur ce sujet soulèvent une question plus fondamentale sur la manière dont ils sont passés des données particulières qu'ils ont recueillies à leurs conclusions plus générales. Ils nous disent maintenant que des individus comme Omer B. étaient, d'une certaine manière, «représentatifs» d'une population plus vaste. Mais de quelle population parlent-ils et quelles preuves apportent-ils pour appuyer leur affirmation à l'effet que ces directeurs étaient «typiques»? Ces questions me ramènent à mon compte rendu où j'avais critiqué leur manière un peu trop cavalière d'utiliser des termes comme «groupe témoin» et «échantillon». Leur réponse ne me 
satisfait pas vraiment. Au contraire, ils ont profité de l'occasion pour mettre en doute la méthodologie de mon propre livre sur les caisses populaires dans lequel j'avais examiné les activités de cinq caisses. Toutefois, je n'ai jamais prétendu que ces caisses étaient nécessairement «représentatives» au sens statistique.

$\mathrm{Si}$, sur cette question comme ailleurs, Levasseur et Rousseau répondent à mon compte rendu en critiquant un livre que j'ai écrit il y a six ans, je n'ai pas l'intention d'abuser de la patience des lecteurs en défendant ici In Whose Interest. Les lecteurs intéressés à comparer deux façons très différentes d'aborder l'histoire des caisses peuvent désormais consulter mon livre et celui de Levasseur et Rousseau et tirer leurs propres conclusions sur des enjeux tels que l'utilisation des sources et l'intégration des conclusions dans l'ensemble de la recherche internationale. Ma principale critique au sujet de Du comptoir au réseau financier concernait justement son faible intérêt envers la défense d'un point de vue précis dans le contexte de l'historiographie actuelle sur la question. Si mon compte rendu a incité les auteurs, qui continuent leur recherche sur les caisses populaires, à prendre un virage plus analytique, alors, je m'estime heureux.

Traduction: Marie Poirier 